\title{
Actinobacillus ureae septic arthritis in a returning traveler from Gambia: A case report and a review of literature
}

\author{
B. Soerajja Bhoelan, Marco Goeijenbier*, Carla van Tienen and Lennert Slobbe \\ University Medical Center Utrecht, Utrecht, The Netherlands
}

\begin{abstract}
We present, to our knowledge, the second case of a Actinobacillus ureae septic arthritis in a returning traveller from The Gambia with a past medical history of monoclonal gammopathy of undetermined significance complicated by a severe polyneuropathy of the lower extremities.
\end{abstract}

\section{Background}

Actinobacillus ureae, formerly known as Pasteurella ureae [1], is a non-motile and non-sporing Gram-negative rod [2]. Together with the genera Haemophilus it constitutes the family of Pasteurellaceae [3]. It is rarely reported as a causative infectious micro-organism in humans [46]. A. ureae was first isolated in human sputum in three patients with chronic rhinosinusitis [4]. Later, it was also detected in routine sputum testing in patients without any respiratory symptoms and was therefore believed to be a commensal of the respiratory tract [5].

Symptoms may occur in the presence of damage of the upper respiratory tract or bronchial tree or a compromised immune system [5]. Arthritis caused by A. ureae is even more rare, as only one single case has been described in the literature [7]. Here, we report the second published case of septic arthritis due to $A$. ureae, with a possibly tropical origin.

\section{Case report}

A 67-year-old man presented at the emergency department, twentyfour hours after returning from a fourteen-day holiday to The Gambia. He suffered from a swollen, red and painful left foot, which had started three days before admission. The day before admission the patient also had developed fever up to 39 degrees Celsius. In The Gambia, he had resided in several hotels and resorts. He had received adequate travelling immunization and had used atovaquone/proguanil as malaria prophylaxis. His past medical history revealed a polyneuropathy caused by a monoclonal gammopathy of undetermined significance (MGUS), resulting in frequently occurring wounds on the soles of his feet. In The Gambia, he mentioned to have walked barefoot almost constantly. At presentation, no wounds were noticed at the affected foot, apart from a partially removed nail of the first digit.

His vital signs comprised a body temperature of 39.2 degrees Celsius and a tachycardia of $118 / \mathrm{min}$ with a blood pressure of $110 / 68$ mmHg. Physical examination confirmed warmth and swelling of the left foot, mainly located around the ankle as can be seen in figure 1, without evident loss of motion. Laboratory testing revealed elevated inflammation parameters (C-reactive protein of $189.9 \mathrm{mg} / \mathrm{L}$ and white blood cell count of $14.5^{\star} 10^{\wedge} 9 / \mathrm{L}$ ) (Figure 2$)$. No other abnormalities were detected. Blood cultures were taken.
Due to the severity of infection, he was hospitalized to be treated with flucloxacillin $1000 \mathrm{mg}$ four times a day intravenously under the suspicion of a cellulitis. The next day, he had increasing loss of motion of the affected ankle with worsening pain and swelling. Arthritis was confirmed after aspiration of purulent fluid by an ultrasound guided diagnostic synovial puncture. Furthermore, the blood cultures revealed growth of gram-negative rods. At this point the antibiotics were switched to ceftriaxone $2000 \mathrm{mg}$ once a day intravenously.

Both the cultures of blood and synovial aspirate eventually revealed the presence of $A$. ureae, determined by matrix-assisted laser desorption/ionization time-of-flight analyzer (MALDI-TOF, Bruker). An overview of antibiotics susceptibility testing is given in table 1 .

Antibiotic regimen was narrowed to benzylpenicillin $1 \times 10^{\wedge} 6$ IE four times a day intravenously. The infection parameters declined and the body temperature normalized. The pain and loss of motion improved gradually. An arthroscopic irrigation with saline was performed to potentiate bacterial eradication and to restrict intraarticular damage. Repeated scrupulous investigation revealed a wood splinter deep under the skin of the heel of the affected foot, which may potentially have functioned as a portal of entry, although the extracted nail seems to be an alternative legitimate explanation.

The intravenous benzylpenicillin regimen was continued for two weeks. After two weeks, he was discharged from the hospital. Antibiotic treatment was switched to levofloxacin (500mg twice daily) orally for another four weeks. Our patient fully recovered without any sequelae, including normal ankle joint function.

\section{Discussion}

To the best of our knowledge, we reported, the second known case of septic arthritis due to Actinobacillus ureae in a traveler returning from The Gambia. A Medline search was performed using the terms

Correspondence to: M. Goeijenbier, MD, Erasmus Medical Centre, Department of Virology, 's-Gravendijkwal 230, 3015 CE Rotterdam, the Netherlands, Tel: 0031 (0)6-22415084, E-mail: marco.goeijenbier@gmail.com/ marco. goeijenbier@erasmusmc.nl

Received: June 11, 2017; Accepted: July 20, 2017; Published: July 24, 2017 


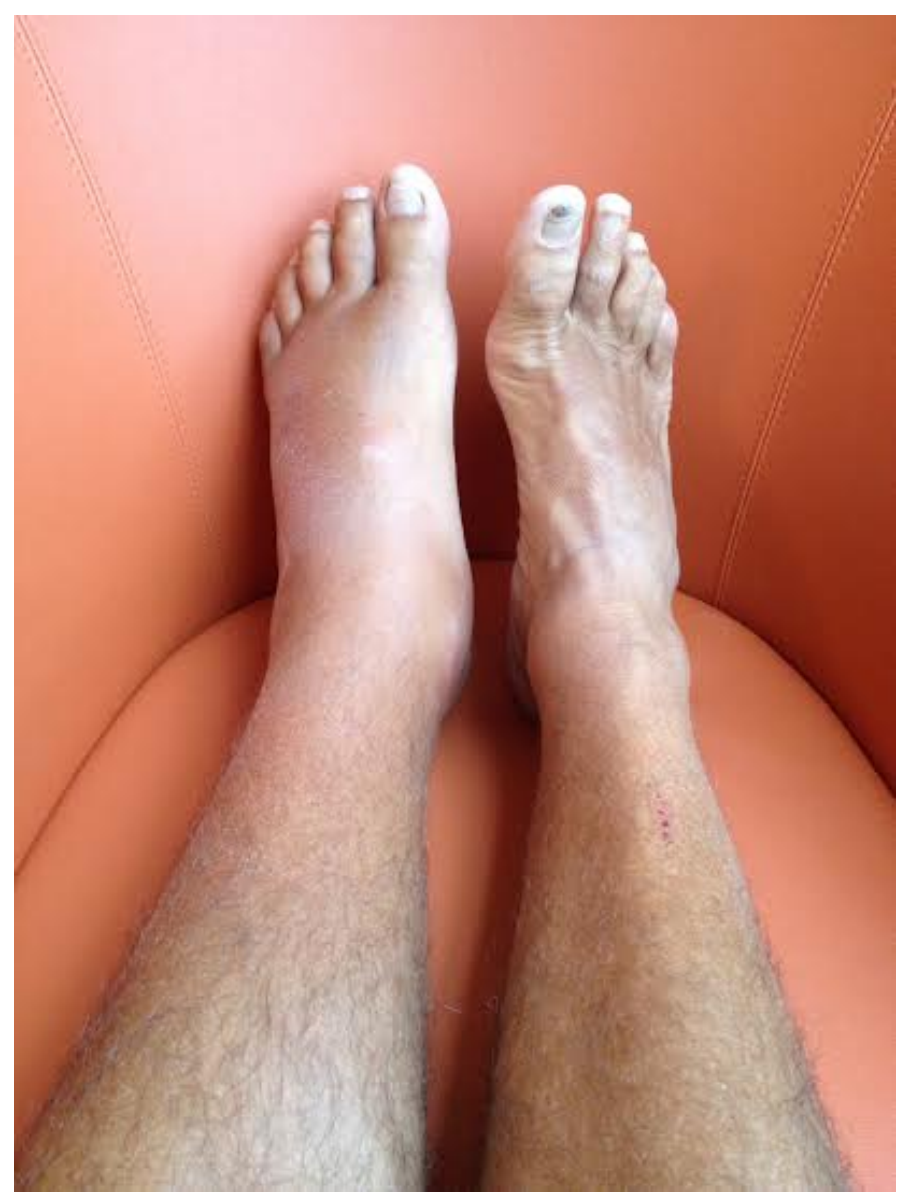

Figure 1. Redness and swelling of the left ankle due to septic arthritis with a damaged first digit's nail

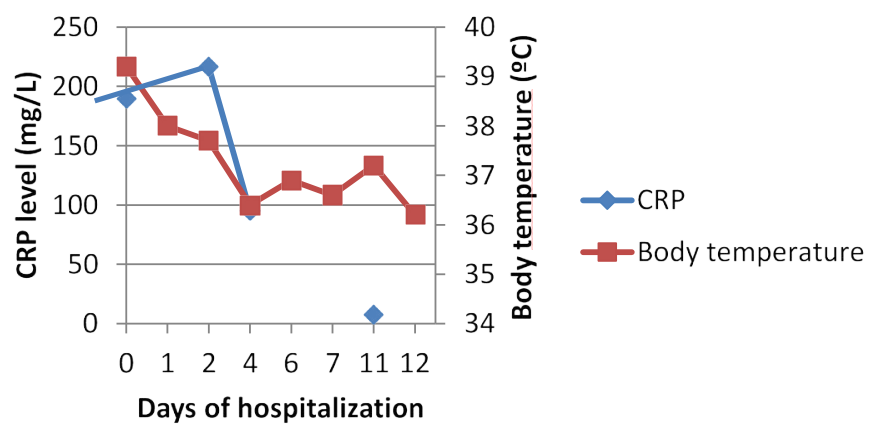

Figure 2. Course of C-reactive protein (CRP) and white blood count (WBC) during hospitalization. Day $0=$ day of admission. Antibiotic switches were made on day one from flucloxacillin to ceftriaxone and on day four to benzylpenicillin. Arthroscopic irrigation took place on the fifth day, which can explain the temporary increase in body temperature

Table 1. Antibiotic susceptibility testing.

\begin{tabular}{|c|c|}
\hline & MIC $(\boldsymbol{\mu g} / \mathbf{m L})$ \\
\hline Penicillin & 0.25 \\
\hline Amoxcillin/clavulanate & 0.125 \\
\hline Meropenem & 0.032 \\
\hline Ceftriaxone & $<0.002$ \\
\hline Ciprofloxacin & 0.032 \\
\hline
\end{tabular}

MIC: Minimum Inhibitory Concentrationa
'Actinobacillus ureae', 'Pasteurella ureae' and 'infection', as this microorganism is uncommonly recognized as a causative infectious agent in humans [4-6]. Twenty-nine cases of infections caused by A. ureae were identified [6-32]. The most frequently reported infection was meningitis. Only one case concerned arthritis [7]. An overview is given in table 2. Interestingly, the case of arthritis of Kaur et al. and our patient both concern $A$. ureae arthritis possibly acquired in Africa. However, no specific associations with the tropics are currently known from literature. Besides, both cases originate from varying demographic locations.

Infections with $A$. ureae seem to be associated with several comorbidities. A substantial part of the meningitis cases was associated with skull fractures or intracranial surgery. Both the current case and the case of arthritis of Kaur et al. concern patients with skin damage. Several patients had a hampered immune system, due to Waldenström's macroglobulinaemia, HIV-positivity and use of immunosuppressive agents. Whether this suggests that disruption of immunity not only predisposes to infection in general, but also to infection with $A$. ureae specifically remains unclear.

The laboratory tests used in this case were CRP, an acute phase protein, and WBC. Both were elevated and in combination with the clinical signs this raised the suspicion of an infection. This was confirmed with bacterial growth in the blood and synovial aspirate cultures.

Routine incubation of the blood and synovial fluid led to rapid identification of the A. ureae. Three blood culture sets (two on the day of admission and one on the second day) were incubated and the aerobic bottles became positive within 14 hours (BACTEC, BD). The synovial fluid grew in the blood culture media and not on the directly inoculated agar, but this could be due to the fact that the synovial fluid was collected after antibiotics were started. In conclusion, the A. ureae grew within 24 hours on blood agar plates and was identified by the MALDI-TOF.

A. ureae can be adequately treated with beta-lactam antibiotics. Alternative options include tetracyclins, sulfonamids/trimethoprim, macrolids and aminoglycosides, which was confirmed from our antibiotic susceptibility tests.

Additionally, in treating septic arthritis it must be considered to perform joint irrigation as to potentiate bacterial eradication and limit intra-articular damage [33]. In both our and the formerly described case of $A$. ureae, residual damage was not reported.

A. ureae seldom is a causative infectious agent of septic arthritis. Currently available literature might suggest an association with disruption of immunity. We recommend to routinely perform blood or synovial cultures in patient with arthritis, which may sometimes detect uncommon pathogens, for which antibiotic susceptibility guided treatment can be initiated.

\section{Consent for publication}

Written informed consent was obtained from the patient for publication of this case report and any accompanying images. 
Table 2. An overview of literature

\begin{tabular}{|c|c|c|c|c|c|c|c|c|}
\hline Case & Year & $\begin{array}{l}\text { Gender } \\
\text { Age }\end{array}$ & Localisation & Relevant history & Antibiotics (AB) & Duration AB & $\begin{array}{l}\text { Additional } \\
\text { therapy }\end{array}$ & Outcome \\
\hline $1[7]$ & 2004 & F, 59 & Arthritis, multifocal & $\begin{array}{c}\text { Rheumatoid arthritis treated } \\
\text { with anti-TNF-alfa and } \\
\text { methotrexate } \\
\text { Trip to Kenya } \\
\text { Several skin abrasions }\end{array}$ & $\begin{array}{l}\text { IV piperacillin/ } \\
\text { tazobactam and } \\
\text { ciprofloxacin } \\
\text { Oral ciprofloxacin }\end{array}$ & 6 weeks & $\begin{array}{c}\text { Wound } \\
\text { debridement } \\
\text { Articular irrigation } \\
\text { Subtotal } \\
\text { synovectomy }\end{array}$ & Survived \\
\hline $2[8]$ & 1968 & M, 2 & Bacteraemia & Malnutrition & N/A & N/A & N/A & Died \\
\hline $3[9]$ & 1996 & M, 65 & Bone marrow & Rheumatoid arthritis & $\begin{array}{l}\text { IV benzylpenicillin } \\
\text { Oral tetracyclin }\end{array}$ & $\begin{array}{l}2 \text { weeks } \\
2 \text { weeks }\end{array}$ & - & Survived \\
\hline $4[10]$ & 1995 & N/A & Bronchitis, chronic & N/A & N/A & N/A & N/A & N/A \\
\hline $5[11]$ & 1981 & N/A & Bronchopneumonia & N/A & N/A & N/A & N/A & N/A \\
\hline $6[12]$ & 1981 & M, 19 & $\begin{array}{c}\text { Bronchopneumonia } \\
\text { Bacteraemia }\end{array}$ & Liver cirrhosis & N/A & N/A & N/A & Died \\
\hline $7[13]$ & 1979 & $\mathrm{~F}, 2$ days & Conjunctivitis & Premature newborn & $\begin{array}{c}\text { Ocular } \\
\text { chloramphenicol }\end{array}$ & N/A & Ocular saline & Full recovery \\
\hline $8[14]$ & 2007 & $\mathrm{~F}, 4$ & Conjunctivitis & - & $\begin{array}{l}\text { amoxicillin/ } \\
\text { clavulanate }\end{array}$ & 5 days & - & Full recovery \\
\hline $9[15]$ & 1993 & M, 59 & Endocarditis & $\begin{array}{c}\text { Previous S. aureus } \\
\text { endocarditis } \\
\text { Periodontal surgery without } \\
\text { AB prophylaxis }\end{array}$ & $\begin{array}{l}\text { IV gentamicin } \\
\text { IV piperacillin } \\
\text { Oral cefotiam }\end{array}$ & $\begin{array}{c}5 \text { weeks } \\
6 \text { weeks } \\
\text { N/A }\end{array}$ & - & Survived \\
\hline $10[16]$ & 1988 & M, 27 months & Otitis media & - & $\begin{array}{l}\text { Oral amoxicillin/ } \\
\text { clavulanate }\end{array}$ & 10 days & - & Full recovery \\
\hline $11[17,18]$ & 1961 & M, 39 & Meningitis & - & N/A & N/A & N/A & Survived \\
\hline $12[19]$ & 1966 & $\mathrm{M}, 48$ & Meningitis & $\begin{array}{l}\text { Alcohol abuse } \\
\text { Skull fracture }\end{array}$ & $\mathrm{N} / \mathrm{A}$ & $\mathrm{N} / \mathrm{A}$ & N/A & Survived \\
\hline $13[20]$ & 1967 & M, 16 & Meningitis & - & N/A & N/A & N/A & Died \\
\hline $14[21]$ & 1978 & F, 53 & $\begin{array}{c}\text { Meningitis } \\
\text { Bacteraemia }\end{array}$ & Intracranial surgery & $\mathrm{N} / \mathrm{A}$ & N/A & $\mathrm{N} / \mathrm{A}$ & Survived \\
\hline $15[22]$ & 1983 & $\mathrm{M}, 40$ & $\begin{array}{l}\text { Meningitis } \\
\text { Endocarditis }\end{array}$ & $\begin{array}{l}\text { Schizophrenia } \\
\text { Alcohol abuse } \\
\text { Odontal infection }\end{array}$ & $\mathrm{N} / \mathrm{A}$ & N/A & $\mathrm{N} / \mathrm{A}$ & Coma \\
\hline $16[23]$ & 1983 & M, 55 & $\begin{array}{l}\text { Meningitis } \\
\text { Bacteraemia }\end{array}$ & Insulin-dependent diabetes & Ampicillin & $\mathrm{N} / \mathrm{A}$ & - & $\begin{array}{c}\text { Survived } \\
\text { Hearing loss }\end{array}$ \\
\hline $17[24]$ & 1983 & M, 54 & Meningitis & $\begin{array}{l}\text { Previous skull fracture } \\
\text { Alcohol abuse }\end{array}$ & Penicillin & N/A & $\mathrm{N} / \mathrm{A}$ & Survived \\
\hline $18[25]$ & 1985 & M, 6 & Meningitis & Previous skull fracture & $\begin{array}{l}\text { IV ampicillin and } \\
\text { chloramphenicol }\end{array}$ & N/A & - & Survived \\
\hline $19[26]$ & 1989 & M, 52 & Meningitis & $\begin{array}{l}\text { Previous skull fracture } \\
\text { Chronic sinusitis }\end{array}$ & ampicillin & 2 weeks & $\mathrm{N} / \mathrm{A}$ & Survived \\
\hline $20[18]$ & 1987 & M, 26 & $\begin{array}{c}\text { Meningitis } \\
\text { Bacteraemia }\end{array}$ & $\begin{array}{c}\text { Alcohol abuse } \\
\text { Two pneumococcal } \\
\text { meningitis in history } \\
\text { Previous skull fracture }\end{array}$ & $\begin{array}{l}\text { IV cefotaxime and } \\
\text { penicillin }\end{array}$ & 8 days & $\begin{array}{l}\text { Neurosurgical } \\
\text { repair of fistula } \\
\text { from lamina } \\
\text { cribrosa and nasal } \\
\text { cavity }\end{array}$ & Survived \\
\hline $21[27]$ & 1994 & $\mathrm{M}, 25$ & Meningitis & $\begin{array}{l}\text { HIV-positive } \\
\text { Head trauma }\end{array}$ & $\begin{array}{l}\text { IV ceftriaxone } \\
\text { switched to IV } \\
\text { penicillin }\end{array}$ & $\mathrm{N} / \mathrm{A}$ & - & Survived \\
\hline $22[28]$ & 1995 & $\mathrm{M}, 17$ & Meningitis & $\begin{array}{l}\text { Skull fracture } \\
\text { Dural tears }\end{array}$ & $\begin{array}{l}\text { IV penicillin and } \\
\text { ceftazidime }\end{array}$ & N/A & $\begin{array}{l}\text { Frontal craniotomy } \\
\text { with partial } \\
\text { debridement left } \\
\text { frontal lobe } \\
\text { Repair dural tears }\end{array}$ & $\begin{array}{l}\text { Survived } \\
\text { Complete } \\
\text { neurological } \\
\text { recovery }\end{array}$ \\
\hline $23[29]$ & 2002 & M, 22 & Meningitis & $\begin{array}{l}\text { Previous neurosurgery } \\
\text { Skull fracture }\end{array}$ & IV ceftriaxone & 10 days & - & survived \\
\hline $24[17]$ & 2009 & M, 75 & Meningitis & $\begin{array}{c}\text { Waldenström's } \\
\text { macroglobulinaemia }\end{array}$ & $\begin{array}{l}\text { IV cefotaxime } \\
\text { Oral amoxicillin }\end{array}$ & $\begin{array}{l}15 \text { days } \\
1 \text { week }\end{array}$ & - & Survived \\
\hline $25[30]$ & 1978 & M, 14 & $\begin{array}{l}\text { Meningo- } \\
\text { encephalitis }\end{array}$ & $\begin{array}{c}\text { Previous basal skull fracture } \\
\text { Dural tear }\end{array}$ & IV ampicillin & N/A & - & Survived \\
\hline $26[6]$ & 1989 & $\mathrm{M}, 44$ & Peritonitis & $\begin{array}{l}\text { Alcohol abuse } \\
\text { Liver cirrhosis } \\
\text { Denver shunt }\end{array}$ & $\begin{array}{l}\text { IV clindamycin } \\
\text { IV ampicillin and } \\
\text { gentamicin }\end{array}$ & $\begin{array}{l}5 \text { days } \\
10 \text { days }\end{array}$ & - & Survived \\
\hline $27[31]$ & 1976 & $\mathrm{M}, 47$ & Pneumonia & $\begin{array}{l}\text { Previous alcohol abuse } \\
\text { Emphysema } \\
\text { Multiple rib fractures }\end{array}$ & N/A & $\mathrm{N} / \mathrm{A}$ & - & Died \\
\hline $28[32]$ & 2000 & $\mathrm{M}, 28$ & Pneumonia & $\begin{array}{c}\text { AIDS } \\
\text { Hepatitis type C }\end{array}$ & IV ceftriaxone & 10 days & - & Survived \\
\hline $29[17,18]$ & 1984 & M, 47 & Septicaemia & $\begin{array}{l}\text { Alcohol abuse } \\
\text { Liver cirrhosis }\end{array}$ & N/A & N/A & $\mathrm{N} / \mathrm{A}$ & Died \\
\hline
\end{tabular}




\section{References}

1. Mutters R, Pohl S, Mannheim W (1986) Transfer of Pasteurella ureae Jones 1962 to Genus Actinobacillus Brumpt 1910: Actinobacillus ureae comb. Int J Syst Bacteriol 36: $343-344$.

2. Bercovier H, Escande F, Grimont PAD (1984) Biological characterization of Actinobacillus species and Pasteurella ureae. Ann Microbiol (Paris) 135: 203-218. [Crossref]

3. Brondz I, Olsen I, Sjöström M (1990) Multivariate analysis of quantitative chemical and enzymic characterization data in classification of Actinobacillus, Haemophilus and Pasteurella spp. J Gen Microbiol 136: 507-513.

4. OMLAND T, HENRIKSEN SD (1961) Two new strains of Pasteurella haemolytica var. ureae isolated from the respiratory tract. Acta Pathol Microbiol Scand 53: 117-120. [Crossref]

5. JONES DM, O'CONNOR PM (1962) Pasteurella haemolytica var. ureae from human sputum. J Clin Pathol 15: 247-248. [Crossref]

6. Noble RC, Marek BJ, Overman SB (1987) Spontaneous bacterial peritonitis caused by Pasteurella ureae. J Clin Microbiol 25: 442-444. [Crossref]

7. Kaur PP, Derk CT, Chatterji M, Dehoratius RJ (2004) Septic arthritis caused by Actinobacillus ureae in a patient with rheumatoid arthritis receiving anti-tumor necrosis factor-alfa theraphy. Rheumatology 31: 1663-1665. [Crossref]

8. Gatti F, Seynhaeve V, Weaver R (1968) [1st description of a case of human septicemia due to Pasteurella ureae]. Ann Soc Belges Med Trop Parasitol Mycol 48: 463-468. [Crossref]

9. Avlami A, Papalambrou C, Tzivra M, Dounis E, Kordossis T (1997) Bone marrow infection caused by Actinobacillus ureae in a rheumatoid arthritis patient. $J$ Infect 35 : 298-299. [Crossref]

10. Vay C, Rodriguez C, Sadorin R, Vujachic P, Famiglietti A (1995) Actinobacillus ureae isolated from a patient with chronic bronchitis. Enferm Infecc Microbiol Clin 13: 569570. [Crossref]

11. Yoshizaki E, Kamiki T, Sakazaki R, Tamura K (1981) A case of bronchopneumonia possibly caused by Pasteurella ureae. Kansenshogaku Zasshi 55: 534-536. [Crossref]

12. Maritz FJ, Franco MM, Swart WH (1981) Pasteurella ureae septicaemia. A case report. S Afr Med J 59: 53-54. [Crossref]

13. Bogaerts J, Lepage P, Kestelyn P, Vandepitte J (1985) Neonatal conjunctivitis caused by Pasteurella ureae. Eur J Clin Microbiol 4: 427-428. [Crossref]

14. Ergin C, Kaleli I, Kiliç I (2007) Acute conjunctivitis caused by Actinobacillus ureae. Pediatr Int 49: 412-413. [Crossref]

15. Yamamoto K, Ikeda U, Ogawa C, Fukazawa H, Eto M, et al. (1993) Pasteurella ureae endocarditis. Intern Med 32: 872-874. [Crossref]

16. Bigel ML, Berardi-Grassias LD, Furioli J (1988) Isolation of Actinobacillus urea (Pasteurella ureae) from a patient with otitis media. Eur J Clin Microbiol Infect Dis 7: 206-207. [Crossref]
17. De Castro N, Pavie J, Lagrange-Xélot M, Bouvri D, Delisle F et al. (2007) Severe Actinobacillus ureae meningitis in an immunocompromised patient: Report of one case and review of the literature. Scand J Infect Dis 39: 1076-1079. [Crossref]

18. Verhaegen J, Verbraeken H, Cabuy A, Vandeven J, Vandepitte J (1988) Actinobacillus (formery Pasteurella) ureae meningitis and bacteraemia: report of a case and review of literature. $J$ Infect 17: 249-253. [Crossref]

19. Wang WL, Haiby G (1966) Meningitis caused by Pasteurella ureae. Am J Clin Pathol 45: 562-565. [Crossref]

20. Thibault P, Robin LA, Magard H, Second L (1967) Fulminating meningitis due to urea variety Pasteurella hemolytica. Presse Med 75: 345-346.

21. Bia F, Marier R, Collins WFJ, von Graevenitz A (1978) Meningitis and bacteremia caused by Pasteurella ureae: Report of a case following intracranial surgery. Scand $J$ Infect Dis 10: 251-253. [Crossref]

22. Brass EP, Wray LM, McDuff T (1983) Pasteurella ureae meningitis associated with endocarditis. Report of a case and review of the literature. Eur Neurol 22: 138-141. [Crossref]

23. Grewal P, Fonseca K, Andrews HJ (1983) Pasteurella ureae meningitis and septicaemia. J Infect 7: 74-76. [Crossref]

24. Marriott DJ, Brady LM (1983) Pasteurella ureae meningitis. Med J Aust 2: 455-456. [Crossref]

25. Yagupsky P, Simo A, Gorodischer R (1985) Pasteurella ureae meningitis as complication of skull fractures. Eur J Clin Microbiol 4: 589-590. [Crossref]

26. Morlat P, Duthil B, Gin H, Ragnaud JM, Aubertin J, et al. (1989) [Actinobacillus ureae meningitis. Apropos of a case]. Pathol Biol (Paris) 37: 1099-1101. [Crossref]

27. Kaka S, Lunz R, Klugman KP (1994) Actinobacillus (Pasteurella) ureae meningitis in a HIV-positive patient. Diagn Microbiol Infect Dis 20: 105-107. [Crossref]

28. Kingsland RC1, Guss DA (1995) Actinobacillus ureae meningitis: case report and review of the literature. J Emerg Med 13: 623-627. [Crossref]

29. Whitelaw AC, Shankland IM, Elisha BG (2002) Use of 16S rRNA sequencing for identification of Actinobacillus ureae isolated from a cerebrospinal fluid sample. J Clin Microbiol 40: 666-668. [Crossref]

30. Kolyvas E, Sorger S, Marks MI, Pai CH (1978) Pasteurella ureae meningoencephalitis. J Pediatr 92: 81-82. [Crossref]

31. Starkebaum GA, Plorde JJ (1977) Pasteurella pneumonia: report of a case and review of the literature. J Clin Microbiol 5: 332-335. [Crossref]

32. Perez JA, De la Iglesia A, de la Iglesia M, Menchero A (2000) Pneumonia caused by Actinobacillus ureae Enfermedades infecciosas y microbiologia clinica 18: 296-297.

33. Mathews CJ, Kingsley G, Field M, Jones A, Weston VC, et al. (2007) Management of septic arthritis: a systematic review. Ann Rheum Dis 66: 440-445. [Crossref]

Copyright: (C2017 Bhoelan BS. This is an open-access article distributed under the terms of the Creative Commons Attribution License, which permits unrestricted use, distribution, and reproduction in any medium, provided the original author and source are credited. 